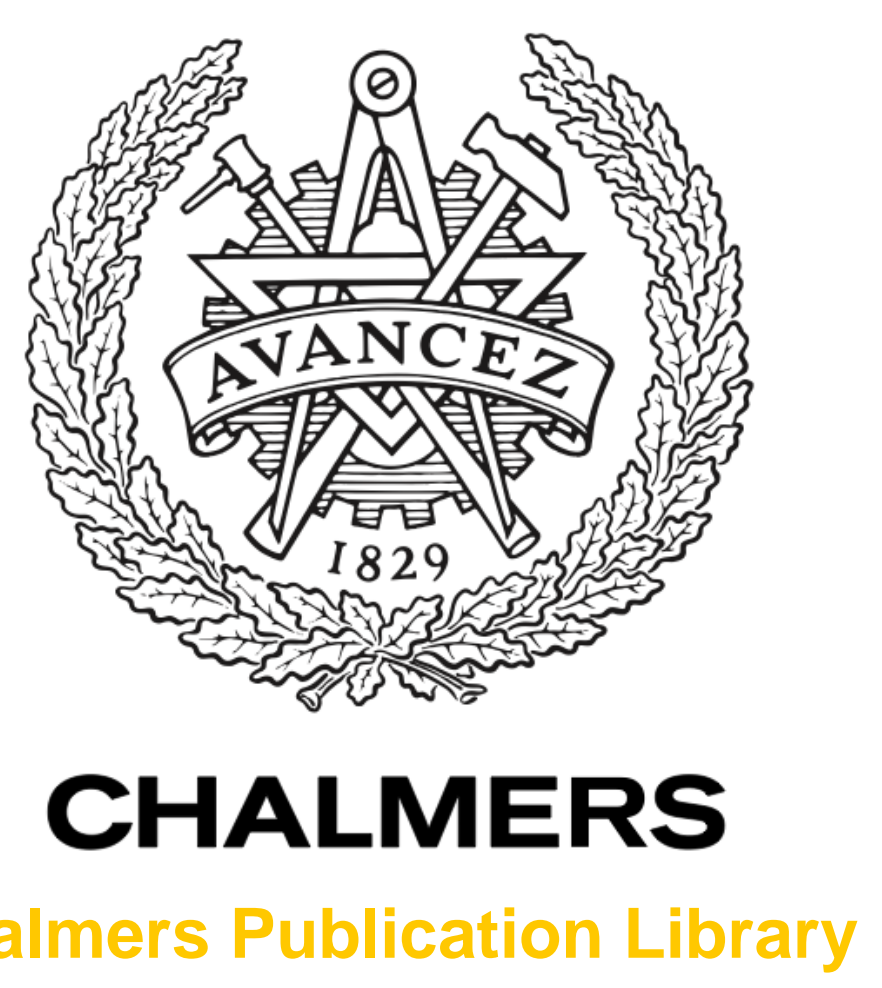

Chalmers Publication Library

\title{
An ultrawideband microwave medical diagnostic system: Design considerations and system performance
}

This document has been downloaded from Chalmers Publication Library (CPL). It is the author's version of a work that was accepted for publication in:

Proceedings of 6th European Conference on Antennas and Propagation, EuCAP 2012. Prague, 26-30 March 2012

Citation for the published paper:

Zeng, X. ; Fhager, A. ; Persson, M. (2012) "An ultrawideband microwave medical diagnostic system: Design considerations and system performance". Proceedings of 6th European Conference on Antennas and Propagation, EuCAP 2012. Prague, 26-30 March 2012 pp. 3664-3668.

http://dx.doi.org/10.1109/EuCAP.2012.6206181

Downloaded from: http://publications.lib.chalmers.se/publication/160307

Notice: Changes introduced as a result of publishing processes such as copy-editing and formatting may not be reflected in this document. For a definitive version of this work, please refer to the published source. Please note that access to the published version might require a subscription. 


\title{
An Ultrawideband Microwave Medical Diagnostic System: Design Considerations and System Performance
}

\author{
Xuezhi Zeng*, Andreas Fhager*, Mikael Persson*, Peter Linner ${ }^{\dagger}$, and Herbert Zirath ${ }^{\dagger}$ \\ * Department of Signals and Systems \\ Chalmers University of Technology \\ 41296 Gothenburg, Sweden. \\ Email: xuezhi@chalmers.se \\ ${ }^{\dagger}$ Microwave Electronics Laboratory \\ Chalmers University of Technology \\ 41296 Gothenburg, Sweden.
}

\begin{abstract}
We discuss several issues in the design of an ultrawideband microwave system dedicated to medical diagnostics. Based on the discussion, a FPGA-based time domain microwave diagnostic system is proposed. The noise sources of the system are identified and the system noise performance is analyzed. As an example, a 2-D antenna system is considered and the measurement signal to noise ratios are evaluated.
\end{abstract}

\section{INTRODUCTION}

Microwave technology has shown its great potential in the diagnostic of tissue abnormalities, i.e. tumor and bleeding [1]-[4]. In this technique, microwave signals are used to illuminate biological tissues (bodies) and the scattered signals are recorded. By utilizing the received data, the tissue abnormalities are identified.

Ultra-wideband(UWB) technology is very attractive in microwave diagnostics. Target response to microwave excitation is dependent on the illuminating frequency, therefore more features about the target can be obtained with UWB illumination compared to a monofrequency case. In microwave imaging, UWB technology allows a good trade off between a fine resolution and an adequate penetration depth in biological tissues.

UWB measurements are usually carried out by using commercial instruments, i.e. a vector network analyzer (VNA)[2] or a pulse generator plus an oscilloscope [5]. The former uses frequency sweeping technique, each time illuminating biological tissues with a different frequency, to obtain the target response over a wideband. The latter uses a UWB pulse generated by the pulse generator to excite tissue and measures the response with the oscilloscope.

However, the above mentioned systems are massive and expensive, therefore not applicable for clinical trials. Therefore, a custom designed UWB microwave system dedicated to medical diagnostics is of great need. The system is expected to have compact size, high measurement speed and low cost.

In this paper, several issues involved in the design of such a system are discussed. Considering the system requirements

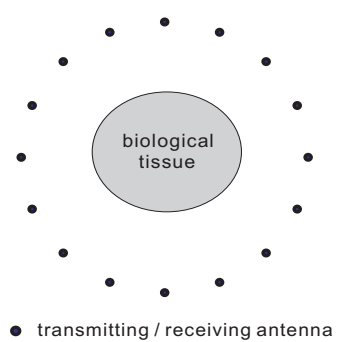

Fig. 1. The measurement configuration of a microwave diagnostic system

and available technologies, we propose a new system design. The system is described and its performance is evaluated.

\section{MicROWAVE MEDICAL DIAGNOSTICS}

Fig. 1 shows the simplified measurement configuration of a microwave system for medical diagnostics.

In the above measurement configuration, biological issue with unknown dielectric properties is surrounded by an antenna array. Each time, a microwave signal is transmitted by one of the antennas into the tissue, and the scattered field is acquired by the remaining antennas. This process is repeated until all the antennas have been used for transmitting. The received signals are processed either to give some characteristic parameters related to the tissue's features or to produce an image of the dielectric property distribution [3]. The microwave signal can be a monofrequency, multiplefrequency or UWB signal. Our work focus on UWB data.

\section{DESIGN CONSIDERATIONS}

Several factors in the design of a UWB microwave system for medical applications are discussed in the following.

\section{A. Frequency range of interest}

There are two main factors need to be considered in choosing an appropriate frequency range. One is the penetration 
depth. Biological tissues are generally lossy, therefore a higher frequency has a lower penetration depth in tissues, which might result in a miss-detection of internal tissue abnormalities due to the inadequate microwave illumination. From this point of view, a lower frequency is preferred. The other factor is the spatial resolution, which is a very important performance for microwave imaging. It is well known that a higher frequency gives a finer resolution.

As a result, these two factors are contradictory to each other in choosing the frequency range. According to our previous numerical studies [2] and the hardware available on the market, we choose the frequency range $500 \mathrm{MHz}-4 \mathrm{GHz}$ as a trade-off.

\section{B. Measurement technology}

As mentioned in the introduction section, UWB measurements can be performed either in the frequency domain or in the time domain. In the frequency domain, a large number of measurements need to be performed on different discrete frequencies, which is very time consuming. In comparison with frequency domain technology, time domain measurement technology is more promising due to the fast measurement and simple system structure.

The generation of UWB pulses with ultra-short duration(less than hundred picosecond) has become possible due to the advancement of solid-state technology. UWB pulse generation is usually based on step recovery diode (SRD) or nonlinear transmission line (NLTL) methods. SRD method is simple and inexpensive, but have a moderately high level of random jitter. NLTL-based pulse generators generally have lower jitter, but are more complex to manufacture, and are not suited for minimization.

\section{Data acquisition technique}

The data acquisition of a time domain signal mainly includes sampling and digitization. Two main parameters, analog bandwidth and sampling rate, need to be considered in this process. The analog bandwidth of the data acquisition module should cover the frequency range of interest in order to reduce signal reflections due to electrical mismatch. According to Nyquist sampling theorem, the sampling rate should be at least twice of the signal bandwidth in order to reconstruct the original signal.

Due to the lack of an A/D converter with an adequate analog bandwidth and a real time sampling rate, the sampling and digitizing of a UWB signal is accomplished by using a wideband sample and hold component ahead of a lower speed A/D converter. The sample and hold component provides an adequate analog bandwidth, and an equivalent time sampling technique is employed to give a sufficient effective sampling rate.

\section{PRoposed DESIGN}

In this section, the proposed system design is described. Noise sources are identified and the noise power from different parts are analyzed.

\section{A. System Architecture}

Fig. 2 presents the custom-designed UWB system. It is composed of a pulse generator, a pair of transmitting-receiving antennas, a low noise amplifier (LNA), a track and hold amplifier $(\mathrm{T} / \mathrm{H})$, an analog and digital converter(ADC), and a FPGA board.

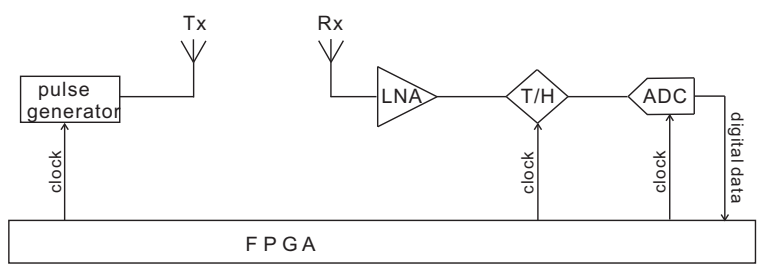

Fig. 2. The block diagram of the custom-designed UWB system.

The system operation can be summarized as below: a UWB pulse generated by the pulse generator is radiated by the transmitting antenna to illuminate an inspected object and the scattered signal is acquired by the receiving antenna. The received signal is amplified with the LNA and subsequently sampled and digitized by the $\mathrm{T} / \mathrm{H}$ and $\mathrm{ADC}$. The whole measurement process is triggered and controlled by the FPGA board.

Ideally, the amplified UWB signal is directly fed to a wideband, high dynamic range ADC for digitization. Key limitations to this approach is that current ADCs do not usually have sufficient bandwidth and sampling rate for these very wideband applications. In addition, maintenance of good sampling linearity at frequencies above the UHF band is technologically challenging and most current ADCs suffer rapidly degrading linearity above one or two $\mathrm{GHz}$ signal frequency. These limitations can be overcome by using a UWB $\mathrm{T} / \mathrm{H}$ ahead of an ADC. The received signal is sampled with the $\mathrm{T} / \mathrm{H}$ and the output will keep constant for a period of time. The low bandwidth held output waveform can be processed by an ADC with substantially reduced bandwidth.

According to the frequency range of interest, pulse generator Model 3500D from Picosecond-Pulse-Lab(PSPL) is chosen for UWB signal generation. The pulse generator has a tunable output voltage and repetition rate, therefore gives high flexibility for the system design [7]. A LNA and T/H from Hittite microwave are chosen for ensuring good performance in the frequency range of interest with a low cost [8]. In order to facilitate the system development, an ADC from Texas instrument is selected [9].

\section{B. Noise sources}

Noise present in the system can be classified as vertical noise and horizontal noise. Here we only consider vertical noise. The noise present in the vertical axis includes the external noise intercepted by the antenna, the thermal noise generated by the LNA and the noise contributed by the sampling and digitizing (T/H plus ADC) module.

Referring to the sampling interface, the noise power contributed by the antenna can be expressed by the following 
equation:

$$
n_{a}=\int_{f_{1}}^{f_{2}} K_{B} \cdot T_{a} \cdot G(f) \cdot\left|H_{s}(f)\right|^{2} d f
$$

where $f_{1}$ and $f_{2}$ define the antenna bandwidth, $K_{B}=$ $1.38066 \times 10^{-23} \mathrm{~J} / \mathrm{K}$ is the Boltzmann constant, $T_{a}=290 \mathrm{~K}$ is the antenna noise temperature, $G$ is the power gain of the LNA and $H_{s}$ is the sampling transfer function, which behaves as a low-pass filter:

$$
H_{s}(f)=\frac{1}{1+j\left(f / f_{b}\right)}
$$

where $f_{b}=4.5 \mathrm{GHz}$ is the bandwidth of the $\mathrm{T} / \mathrm{H}$ [10].

In a similar manner, the noise power contributed by the LNA can be obtained from the following:

$$
n_{f}=\int_{0}^{\infty} K_{B} \cdot[N F(f)-1] T_{0} \cdot G(f) \cdot\left|H_{s}(f)\right|^{2} d f
$$

where $N F$ is the noise figure of the LNA and $T_{0}=290 \mathrm{~K}$ is the room temperature.

If neglecting the mismatches effect, the total system noise power, $n_{\text {sys }}$, can be obtained from the following equation:

$$
n_{s y s}=n_{a}+n_{f}+n_{t h}+n_{a d c}
$$

Here $n_{t h}$ and $n_{a d c}$ are the noise power contributed by the $\mathrm{T} / \mathrm{H}$ and the ADC respectively.

\section{SYSTEM PERFORMANCE}

Signal to noise ratio (SNR) is the most important performance metric for UWB microwave diagnostic systems. Poor SNRs may result in miss-detection or distortions to the image reconstructions [6].

\section{A. SNR definition}

For a power signal, e.g, a sinusoidal signal, SNR is usually defined as the ratio between the signal power and noise power. This definition however, does not fits to the UWB microwave diagnostic system since a UWB pulse is not a power signal but an energy signal. Therefore, a SNR definition made in frequency domain is used to characterize the performance of the UWB system:

$$
S N R(f)=\frac{S(f)}{N_{\text {sys }}(f)}
$$

where $S(f)$ is the power spectral density of a noise-free sampled signal and $N_{\text {sys }}$ is the noise power density.

The power spectral density of the noise free signal can be calculated from the following equation:

$$
S(f)=\left|V_{s}(f) \cdot T R(f)\right|^{2} \cdot G(f) \cdot\left|H_{s}(f)\right|^{2}
$$

Here, $V_{s}$ is the frequency spectrum of the generator's output pulse, and $T R$ is the transmission coefficient between the transmitting and receiving antennas.

In an equivalent time sampling system, the noise present at the system output does not retain the physical frequency spectrum. Therefore, after splicing all the samples together,

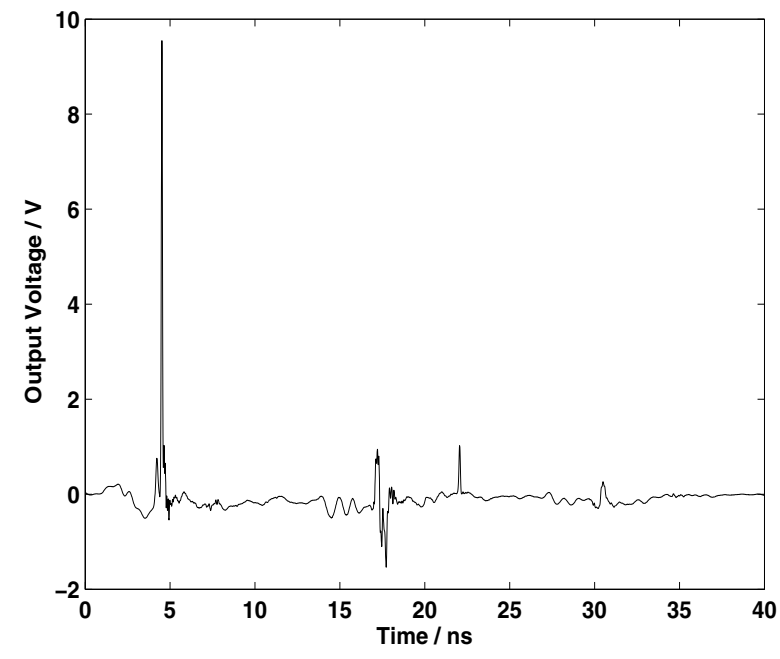

(a)

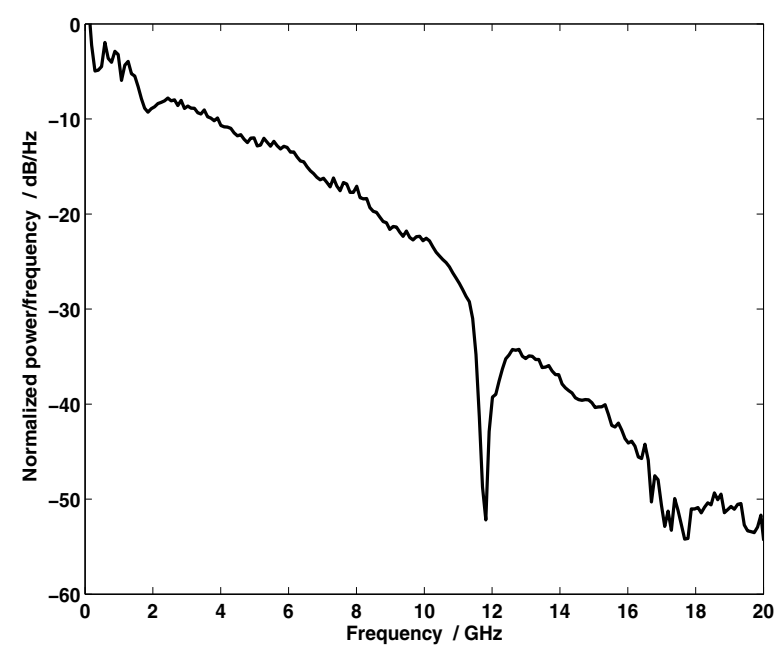

(b)

Fig. 3. The output signal of the impulse generator: (a) maximum output time domain waveform and (b) power spectrum.

the noise power is distributed over the first Nyquist interval and the noise power density $N_{\text {sys }}$ is:

$$
N_{s y s}(f)=\frac{n_{s y s}}{f_{s} / 2}
$$

where $f_{s}$ is the effective sampling rate.

\section{B. Results}

In this section, we evaluate the SNRs of the custom designed microwave diagnostic system according to the above analysis.

The output signal of the impulse generator is recorded by using a $50 \mathrm{GHz}$ sampling oscilloscope and Fig. 3 gives the maximum output signal and the unitary power spectrum. The gain and noise figure of the LNA are obtained by using a noise figure analyzer and the data are presented in Fig. 4.

The SNR is dependent on the antenna system performance and a 2-D antenna system in our lab is used here for the 


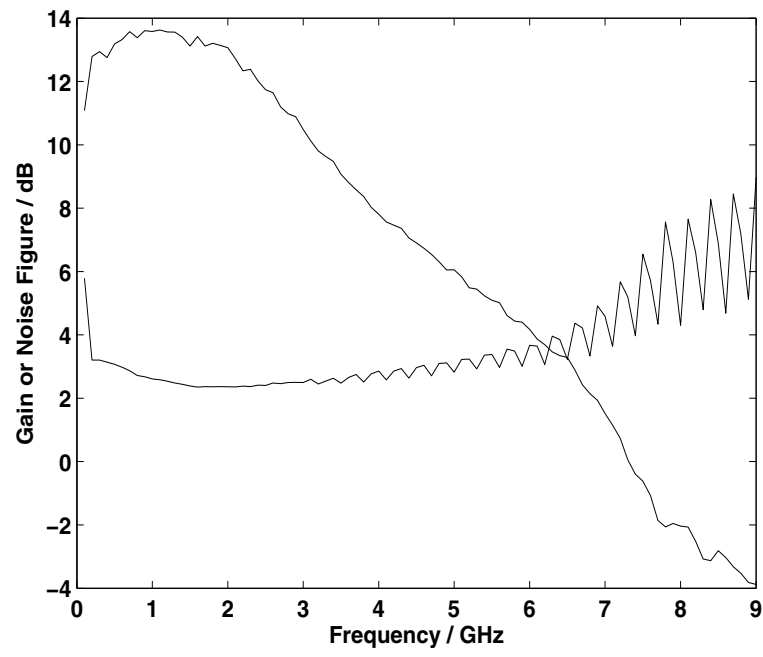

Fig. 4. The power gain and noise figure of the LNA.

evaluation. It is an circular array with twenty monopoles, which are evenly distributed on the bottom metal plane of an rectangular plastic tank. Fig. 5 gives the return loss of one of the antennas in presence with other antennas. Fig. 6 shows transmission coefficients between one antenna to the antennas which are closest and farthest to it respectively.

Based on this data, the noise power contributed by the antenna and the LNA can be obtained from eq. 1 and eq. 3 . According to the antenna return loss presented in Fig. 5, we determine $f_{1}=3 \mathrm{GHz}$ and $f_{2}=5 \mathrm{GHz}$. The noise performance of the $\mathrm{T} / \mathrm{H}$ is given by the manufacturer and it consists of input sampled buffer noise and output amplifier noise. The input sampled buffer noise is "frozen" into samples and is not restrained by the bandwidth of the ADC, while the output amplifier noise contribution is affected by the ADC's bandwidth. The 12-bit ADC from Texas instrument has an analog bandwidth of $800 \mathrm{MHz}$ and the vertical noise power of the ADC, which is composed of thermal noise and quantization noise, can be calculated from its SNR specification data [9]. Table I lists the noise power contributed by different system parts and it can be seen that the $\mathrm{T} / \mathrm{H}$ noise dominates the system noise.

Based on the transmission coefficients present in Fig. 6, the power density of the corresponding sampled noise-free signals can be obtained according to eq. 6. The resulted SNRs are calculated from eq. 7 and are shown in Fig. 7. An effective sampling rate of $50 \mathrm{GSa} / \mathrm{s}$ is assumed for the calculation.

For the 2-D antenna system considered here, the upper line in Fig 7 represents the maximum SNR can be obtained by using the custom designed system. It is limited by the input limit of the $\mathrm{T} / \mathrm{H}$, which is one volt peak to peak. It is seen that a SNR above $50 \mathrm{~dB}$ can be obtained at frequencies close to $3 \mathrm{GHz}$ and the SNR versus frequency is mainly determined by the transmission coefficient between antennas.

\section{CONCLUSION}

The paper presents a FPGA-based UWB microwave diagnostic system which is suitable for medical clinical trials.

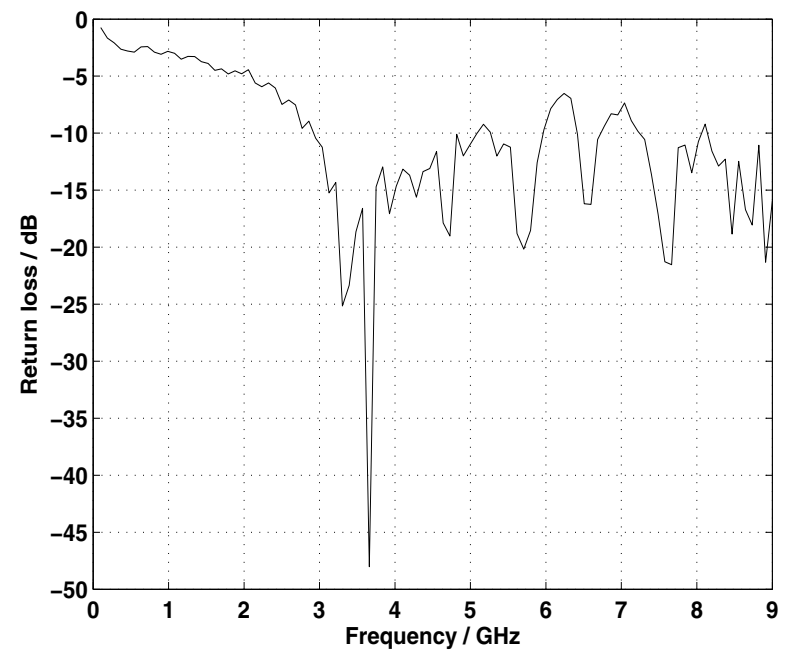

Fig. 5. The return loss of one of the antennas in the antenna array.

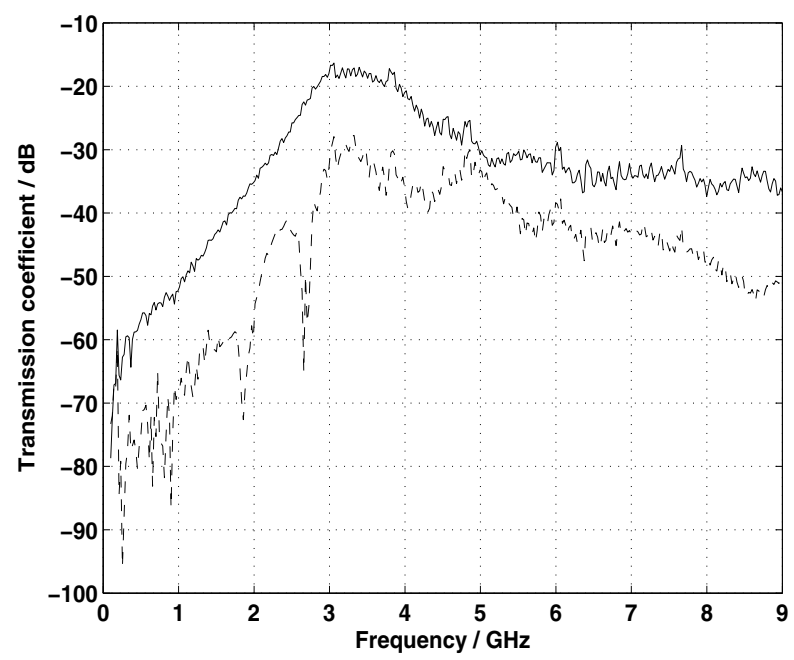

Fig. 6. The transmission coefficients between two antennas. Solid line and dashed line respectively represent the data when the receiving antenna is closest and farthest to the transmitting antenna.

The noise performance of the UWB system is analyzed and the SNRs can be obtained by using the proposed system is evaluated for a specific antenna system. The results show that the $\mathrm{T} / \mathrm{H}$ noise is the main contribution to the system noise power. It is indicated that the SNRs versus frequency largely relies on the transmission coefficient function between antennas.

According to the analysis, the SNRs versus frequency can be improved by increasing the effective sampling rate, which on the other hand takes a longer measurement time. The neglecting of the mismatch effect does not introduce much error in the evaluation since the noise from the antenna and LNA is a very small part of the total system noise.

Only vertical noise is considered in this work and the SNRs due to clock jitter will be discussed in future work. 
TABLE I

SYSTEM NOISE CONTRIBUTION

\begin{tabular}{|l|c|c|c|c|}
\hline Noise Power & $n_{a}+n_{f}$ & $n_{t h}$ & $n_{a d c}$ & $n_{s y s}$ \\
\hline$V^{2} / \mathrm{Hz}$ & $1.2235 \mathrm{e}-08$ & $5.5984 \mathrm{e}-7$ & $4.0653 \mathrm{e}-08$ & $6.1273 \mathrm{e}-07$ \\
\hline
\end{tabular}

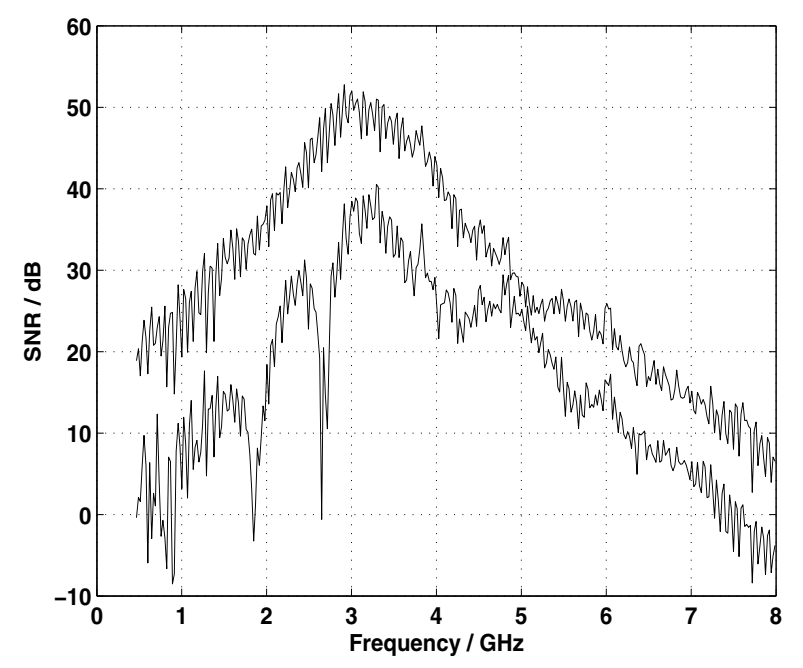

Fig. 7. The SNRs of the signals received by the antennas which are closest (upper line) and farthest (lower line) to the transmitting antenna.

\section{ACKNOWLEDGMENT}

This work was supported in part by VINNOVA within the VINN Excellence Center Chase, and in part by SSF within the Strategic Research Center Charmant.

\section{REFERENCES}

[1] P.M. Meaney, M.W. Fanning, D. Li, S.P. Poplack and K.D Paulsen, "A clinical prototype for active microwave imaging of the breast," IEEE Trans. Microwave Theory Tech., vol. 48, no. 11, pp. 1841-1853, 2000.

[2] A. Fhager, P. Hashemzadeh, and M. Persson, "Reconstruction quality and spectral content of an electromagnetic time-domain inversion algorithm," IEEE Trans. Biomed. Eng., vol. 53, no. 8, pp. 1594-1604, Aug. 2006.

[3] A. Fhager, T. Mckelvey and M. Persson, "Stroke detection using a broad band microwave antenna system," Proceedings of the 4th European Conference on Antennas and Propagation, EUCAP 2010. Barcelona, Spain, 2010.

[4] M. Persson, X. Zeng, and A. Fhager, "Microwave imaging for medical applications," Proceedings of the 5th European Conference on Antennas and Propagation, EUCAP 2011. Rome, 11-15 April 2011.

[5] X. Zeng, A. Fhager, P. Linner, M. Persson and H. Zirath, "Experimental investigation of the accuracy of an ultrawideband time-domain microwave-tomographic System," IEEE Tran. Instrum. Meas., Vol. 60, No. 12, pp. 3939-3949, Dec. 2011.

[6] X. Zeng, A. Fhager, M. Persson, P. Linner and H. Zirath, "Accuracy evaluation of ultra-wideband time domain systems for microwave imaging," IEEE Trans. Antennas Propagat., Vol. 59, No. 11, pp. 4279-4285, Nov. 2011.

[7] Model 3500D Impulse Generator Instruction Manual. Picosecond Pulse Lab.

[8] http://www.hittite.com

[9] ADC Datasheet, http://www.ti.com/lit/ds/symlink/ads41b29.pdf.

[10] http://www.hittite.com/products/view.html/view/HMC660LC4B. 